\title{
Longitudinal Fetal Presentation
}

National Cancer Institute

\section{Source}

National Cancer Institute. Longitudinal Fetal Presentation. NCI Thesaurus. Code C92826.

A type of fetal presentation where the fetal spine is aligned along the longitudinal axis of the uterus and descends into the birth canal during delivery. 